\title{
THE HOTELLING-LAWLEY TRACE STATISTIC FOR CHANGE DETECTION IN POLARIMETRIC SAR DATA UNDER THE COMPLEX WISHART DISTRIBUTION
}

\author{
Vahid Akbari, Stian N. Anfinsen, Anthony P. Doulgeris and Torbjørn Eltoft \\ University of Troms $\varnothing$ \\ Department of Physics and Technology \\ NO-9037 Troms $\varnothing$, Norway
}

\begin{abstract}
In this paper we propose a new test statistic for unsupervised change detection in polarimetric synthetic aperture radar (PolSAR) data. We work with multilook complex (MLC) covariance matrix data, whose underlying model is assumed to be the scaled complex Wishart distribution. We use the complex kind Hotelling-Lawley (HL) trace statistic for measuring the similarity of two covariance matrices. The sampling distribution of the HL trace is approximated by a Fisher-Snedecor distribution, which is used to define the significance level of a constant false alarm rate change detector. The performance of the proposed method is tested on simulated and real PolSAR data sets and compared to the likelihood ratio test statistic.
\end{abstract}

Index Terms - radar polarimetry, synthetic aperture radar, change detection, test statistic, complex Wishart distribution, Hotelling-Lawley trace

\section{INTRODUCTION}

Multitemporal remote sensing represents a powerful source of information to monitor and study processes on the Earth's surface, for instance, in dister management, detection and monitoring of volcanic activity, glacier dynamics, deforestation, and growth of urban areas. The use of synthetic aperture radar (SAR) sensors is attractive in temporal studies, because they are not limited by cloud cover and absence of sunlight, contrary to optical sensors. Many studies have demonstrated the great potential of SAR images in change detection and time series analysis, e.g., $[1,2]$. This study is devoted to the multichannel PolSAR instrument, which provides increased detection capability as compared to single-polarization SAR.

In the analysis of multitemporal SAR data, a large amount of automatic and unsupervised change detection approaches have been developed and described in the literature. We limit our focus to change detection in multilook complex (MLC) PolSAR images, where the backscattered signal is represented by the so-called polarimetric covariance (or coherency) matrix. For each pixel, this is a Hermitian and positive definite matrix, which is often assumed to follow a complex Wishart distribution. The higher complexity of the matrix-variate data makes them less mathematically tractable, which is probably why the literature on incoherent polarimetric change detection is somewhat sparse, and mostly restricted to nontextured density models.

The seminal work on test statistics for change detection in multilook PolSAR images was done by Conradsen et al., who proposed a generalized likelihood ratio test for equality of two complex covariance matrices and gave the asymptotic sampling distribution for the test statistic [3]. This method, also known as the Bartlett test, was compared by Kersten et al. to the alternative contrast ratio and ellipticity tests [4]. Erten et al. presented a test which allows the compared matrices to be statistically dependent [5]. Other methods by Erten et al. based on information theory followed in [6, 7]. Marino et al. proposed a test which is reported to suppress intensity information and perform well in detection of changes in the internal structure of the covariance matrix. All of the mentioned approaches assume that the covariance matrices follow the complex Wishart distribution. Change detection algorithms for non-Wishart PolSAR data described by the multilook product model were presented by Phan et al. [8].

In this study, we apply the complex-kind HotellingLawley (HL) trace as a new test statistic for MLC PolSAR data. The method can be seen as a matrix-variate version of the intensity ratio test, where the HL trace is used to contrast two complex covariance matrices and produce a scalar value, to which a threshold can be applied. Remark that both low and high values of the test statistic indicate change, by analogy with the intensity ratio. The sampling distribution of the proposed test statistic has been approximated by the Fisher-Snedecor (FS) distribution, which has been used as an approximation for the real-kind Hotelling-Lawley trace $[9,10,11]$. The parameters of the FS distribution are determined by matching population moments of the HL trace and the FS distribution, and depend only on the dimension of the polarimetric data and the equivalent number of looks estimated for the images.

Section 2 presents the underlying theory: the complex Wishart distribution, the proposed test statistic and its sampling distribution. Experimental results are given in Section 3 and conclusions in Section 4. 


\section{THEORY}

Let $\mathcal{A}=\{\mathbf{A}(i, j) ; 1 \leq i \leq I, 1 \leq j \leq J\}$ and $\mathcal{B}=$ $\{\mathbf{B}(i, j) ; 1 \leq i \leq I, 1 \leq j \leq J\}$ be two equal-sized and coregistered MLC PolSAR images acquired over the same geographical area at times $t_{0}$ and $t_{1}\left(t_{1}>t_{0}\right)$, where $I$ and $J$ are the number of rows and columns of the images, respectively. To determine whether a change has occured at position $(i, j)$ in the image, we need to compare the PolSAR measurements $\mathbf{A}(i, j)$ and $\mathbf{B}(i, j)$. These are assumed to be statistically independent sample covariance matrices with dimension $d \times d$, where $d$ is the number of polarimetric channels. They are defined on the cone of Hermitian and positive definite matrices, denoted $\Omega_{+}$.

\subsection{Scaled complex Wishart distribution}

The scaled complex Wishart distribution is defined on $\Omega_{+}$and has probability density function (pdf) [12]

$$
p_{\mathbf{C}}(\mathbf{C})=\frac{L^{L d}|\mathbf{C}|^{L-d}}{\Gamma_{d}(L)|\boldsymbol{\Sigma}|^{L}} \exp \left(-L \operatorname{tr}\left(\boldsymbol{\Sigma}^{-1} \mathbf{C}\right)\right)
$$

where $|\cdot|$ is the determinant, $\operatorname{tr}(\cdot)$ is the trace operator, $d$ is the matrix dimension, $L$ is the equivalent number of looks (i.e., the degrees of freedom), $\boldsymbol{\Sigma}=\mathrm{E}\{\mathrm{C}\}$ is the scale matrix, and the normalization factor

$$
\Gamma_{d}(L)=\pi^{\frac{d(d-1)}{2}} \prod_{i=1}^{d} \Gamma(L-i+1)
$$

is the multivariate gamma function of the complex kind. We further assume that both $\mathbf{A}$ and $\mathbf{B}$ (whose pixel indices are dropped from here on) follow complex Wishart distributions, potentially with different distribution parameters, which is denoted $\mathbf{A} \sim \mathcal{W}_{d}^{\mathbb{C}}\left(L_{a}, \boldsymbol{\Sigma}_{a}\right)$ and $\mathbf{B} \sim \mathcal{W}_{d}^{\mathbb{C}}\left(L_{b}, \boldsymbol{\Sigma}_{b}\right)$. For simplicity, we shall assume that images $\mathcal{A}$ and $\mathcal{B}$ have the same original resolution and have been subject to the same multilook processing, which legitimates the assumption of $L_{a}=L_{b}$.

\subsection{Hotelling-Lawley trace statistic}

Under the assumption that $L_{a}=L_{b}$, change detection is performed by choosing between the hypotheses:

$$
\begin{aligned}
& H_{0}: \boldsymbol{\Sigma}_{a}=\boldsymbol{\Sigma}_{b}, \\
& H_{1}: \boldsymbol{\Sigma}_{a} \neq \boldsymbol{\Sigma}_{b} .
\end{aligned}
$$

The null hypothesis $\left(H_{0}\right)$ corresponds to no change and the alternative hypothesis $\left(H_{1}\right)$ to change. We propose to test this with the complex-kind Hotelling-Lawley trace statistic, defined as

$$
\tau_{H L}=\operatorname{tr}\left(\mathbf{A}^{-1} \mathbf{B}\right) .
$$

This measure effectively produces a matrix quotient and compacts the matrices into a scalar, which can be tested against a threshold.
Thresholding can be done with a standard constant false alarm rate (CFAR) procedure, or by alternative methods such as the minimum classification error approach of Kittler and Illingworth (K\&I) $[13,14]$. In the CFAR approach we set a desired false alarm rate (FAR) or significance level $\alpha$, and determine the threshold from the sampling distribution $p_{\tau}(\tau)$ for $\tau_{H L}$ as

$$
\int_{0}^{T_{l o}} p_{\tau}(t) d t=\int_{T_{h i}}^{\infty} p_{\tau}(t) d t=\alpha .
$$

The thesholds $T_{l o}$ and $T_{h i}$ define the lower and the upper $\alpha$ percentiles of $p_{\tau}(\tau)$, and correspond to backscatter decrease and increase, respectively. The HL trace can be used to test for either kind of change, or both, depending on the application. With two thresholds, the definition in (5) must be adjusted such that the two rejection regions together define a FAR equal to $\alpha$.

\subsection{Sampling distribution}

Both the CFAR approach and the K\&I method require knowledge of the sampling distribution of $\tau_{H L}$. It is not mathematically tractable to derive this distribution for general $d$. Hence, we adopt the method used in the real case, which is to approximate $p_{\tau}(\tau)$ by a FS distribution $[9,10,11]$. The motivation is that the FS distribution is the true model for $d=1$, and has generally shown to be an adequate approximation and a practical solution. The pdf is given by [12]

$$
p_{\tau}(t)=\frac{\Gamma(\xi+\zeta)}{\Gamma(\xi) \Gamma(\zeta)} \frac{\xi}{\mu(\zeta-1)} \frac{\left(\frac{\xi}{\mu(\zeta-1)} t\right)^{\xi-1}}{\left(\frac{\xi}{\mu(\zeta-1)} t+1\right)^{\xi+\zeta}}
$$

where $\mu=\mathrm{E}\{\tau\}$ is the mean, while $\xi$ and $\zeta$ are shape parameters. The general $\nu$ th-order moment is

$$
m_{\nu}^{(\mathrm{FS})}\{\tau\}=\left(\frac{(\zeta-1) \mu}{\xi}\right)^{\nu} \frac{\Gamma(\xi+\nu)}{\Gamma(\xi)} \frac{\Gamma(\zeta-\nu)}{\Gamma(\zeta)} .
$$

The remaining problem is to determine the parameters of the FS distribution. Our proposed solution is to match the population moments of the FS distribution, defined by (7), with the population moments of $\tau_{\mathrm{HL}}$. These have been derived in [15] using results from [16]. The mean of $\tau_{H L}$ is

$$
m_{1}^{(\mathrm{HL})}=E\left\{\tau_{\mathrm{HL}}\right\}=\frac{d L_{a}}{Q_{a}} .
$$

where $Q_{a}=L_{a}-d$. The second-order moment is

$$
\begin{aligned}
m_{2}^{(\mathrm{HL})} & =E\left\{\tau_{\mathrm{HL}}^{2}\right\} \\
& =\frac{L_{a}^{2}}{Q_{a}^{3}-Q_{a}}\left(d^{2}\left(Q_{a}+\frac{1}{L_{b}}\right)+d\left(\frac{Q_{a}}{L_{a}}+1\right)\right) .
\end{aligned}
$$




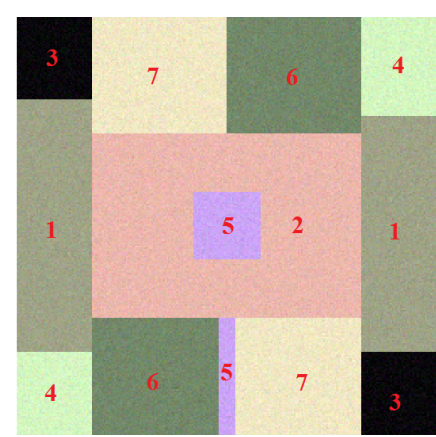

(a) test image 1

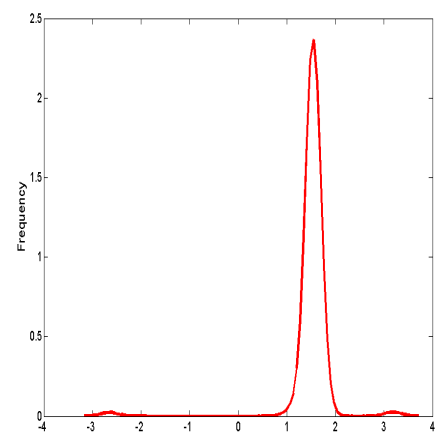

(e) pdf of $\tau_{H L}$

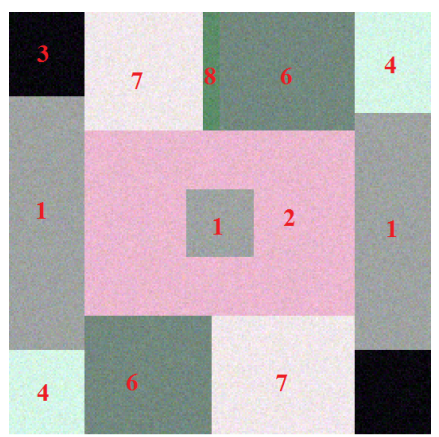

(b) test image 2

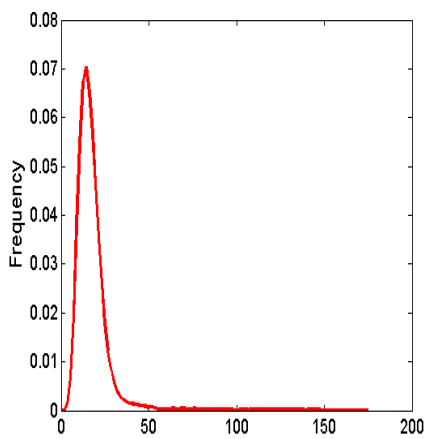

(f) pdf of $\tau_{B}$

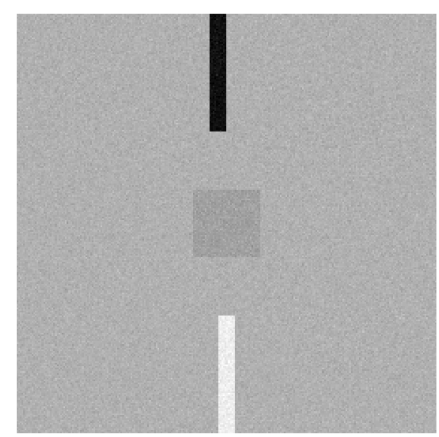

(c) $\log \left(\tau_{H L}\right)$

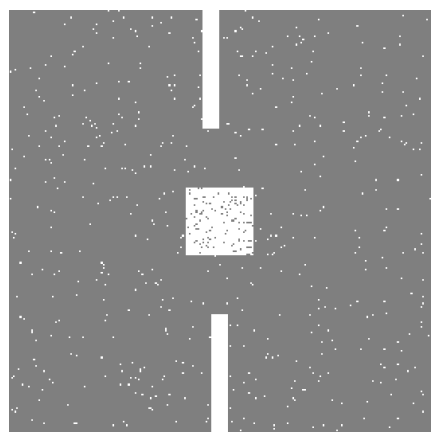

(g) HL test detection result

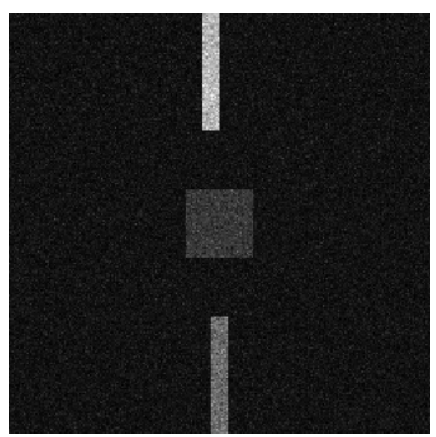

(d) $\log \left(\tau_{B}\right)$

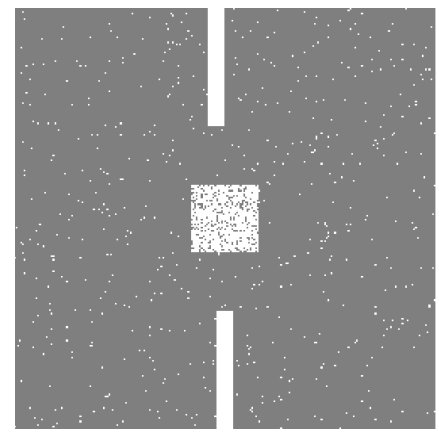

(h) Bartlett test detection result

Fig. 1. Comparison of HL test and Bartlett test with data simulated from the complex Wishart model.

and the third-order moment is given as

$$
\begin{aligned}
& m_{3}^{(\mathrm{HL})}=E\left\{\tau_{\mathrm{HL}}^{3}\right\} \\
& =\frac{L_{a}^{3}}{Q_{a}^{5}-5 Q_{a}^{3}+4 Q_{a}} \times\left[d^{3}\left(\left(Q_{a}^{2}-1\right)+\frac{3 Q_{a}}{L_{b}}+\frac{4}{L_{b}^{2}}\right)\right. \\
& +d^{2}\left(3 Q_{a}+\frac{3\left(Q_{a}^{2}+2\right)}{L_{b}}+\frac{6 Q_{a}}{L_{b}^{2}}\right) \\
& \left.+d\left(4+\frac{6 Q_{a}}{L_{b}}+\frac{2 Q_{a}^{2}}{L_{b}^{2}}\right)\right] \text {. }
\end{aligned}
$$

We seek expressions for the parameters of the FS distribution in terms of the distribution parameters of the scaled Wishart matrices $\mathbf{A}$ and $\mathbf{B}$, i.e., $L=L_{a}=L_{b}$ and $d$. The solutions for $\mu, \xi$ and $\zeta$ are defined by the equation system

$$
m_{\nu}^{(\mathrm{FS})}(\xi, \zeta, \mu)=m_{\nu}^{(\mathrm{HL})}(L, d), \quad \nu=1,2,3 .
$$

An analytic solution for $\mu$ is easily found by matching the first-order moments. To match the second and third-order moments and retrieve the shape parameters, we use minimum distance optimization [17] and minimize

$$
\epsilon^{2}=\sum_{\nu=2}^{3}\left(m_{\nu}^{(\mathrm{HL})}-m_{\nu}^{(\mathrm{FS})}\right)^{2}
$$

Estimation of the equivalent number of looks is a critical point in this procedure. To do this accurately and automatically, we use the unsupervised method by Anfinsen et al. in [18].

\section{RESULTS}

We have performed a number of test on simulated and real data, that will all be presented in a future journal paper. Due to limited space, we here only show an experiment where we have generated two test images with eight classes that follow a scaled complex Wishart distribution with $L=12$ and their individual $\boldsymbol{\Sigma}$ computed from samples of real data. The test images represent times $t_{0}$ and $t_{1}$. They are shown in Fig. 1 (a) and (b) as Pauli images. Here we see that the changes occuring are: (i) a transition from class 1 to class 8 along a vertical strip centered in the upper half of the images; (ii) a transition from class 5 to class 7 in a vertical strip centered in the lower half of the images; (iii) the transition from class 5 to class 1 in the central square. Test statistic images for the proposed HL test $\left(\tau_{H L}\right)$ and the Bartlett test $\left(\tau_{B}\right)$ [3] are respectively shown in (c) and (d) on logarithmic scale to enhance the contrast. The histograms of $\tau_{H L}$ and $\tau_{B}$ follow in (e) and (f), before the change detection results obtained with thresholds set to obtain a CFAR of $1 \%$ are shown in $(\mathrm{g})$ and (h).

A clear difference in the behaviour of the test statistics 
is observed in Fig. 1 (e) and (f), as changes are mapped into the upper tail of $\tau_{b}$, while they can be found in both the upper and the lower tail of $\tau_{H L}$, depending on the nature of the change. Numerical results show that the HL test obtains a superior detection accuracy of $97.9 \%$ against $90.6 \%$ for the Bartlett test, as revealed by Fig. 1 (g) and (h). The measured FAR is $0.90 \%$ for the HL test, while the Bartlett test meets the specified CFAR of $1 \%$ exactly. The overall error rate is $1.02 \%$ for the HL test and $1.40 \%$ for the Bartlett test. We have also produced receiver operating characteristic curves of the two tests for experiments with real data. They unanimously show that the HL test performs better, as it yields a higher detection rate than the Bartlett test when the measured FAR is equal.

\section{CONCLUSIONS}

We have proposed the complex-kind Hotelling-Lawley trace as a test statistic for change detection in multilook PolSAR images. Its sampling distribution has been approximated by a FS distribution, thereby allowing efficient determination of the decision threshold. Experiments show that the proposed test performs better than the commonly used Bartlett test.

\section{REFERENCES}

[1] J. Inglada and G. Mercier, "A new statistical similarity measure for change detection in multitemporal images and its extension to multiscale change analysis," IEEE Trans. Geosci. Remote Sens., vol. 45, no. 5, pp. 14321445, May 2007.

[2] G. Moser and S.B. Serpico, "Unsupervised change detection from multichannel SAR data by Markovian data fusion," IEEE Trans. Geosci. Remote Sens., vol. 47, no. 7, pp. 2114-2128, July 2009.

[3] K. Conradsen, A.A. Nielsen, J. Schou, and H. Skriver, "A test statistic in the complex Wishart distribution and its application to change detection in polarimetric SAR data," IEEE Trans. Geosci. Remote Sens., vol. 41, no. 1, pp. 4-19, Jan. 2003.

[4] P.R. Kersten, J.-S. Lee, and T.L. Ainsworth, "A comparison of change detection statistics in POLSAR images," in Proc. IEEE Int. Geosc. Remote Sens. Symp., IGARSS 2005, Seoul, South Korea, July 2005, vol. 7, pp. 48364839 .

[5] E. Erten, A. Reigber, and R. Zandona-Schneider, "A joint density of interferometric and/or polarimetric images: Application to change detection," in Proc. POLinSAR 2009, Frascati, Italy, vol. ESA SP-668, 3 pp., Apr. 2009.

[6] E. Erten, A. Reigber, and O. Hellwich, "Aspects of multivariate statistical theory with the application to change detection," in Proc. IEEE Int. Geosci. Remote Sens. Symp., IGARSS 2010, Honolulu, USA, 25-30 July 2010, pp. 1960-1963.

[7] E. Erten, O. Chesnokova, C. Rossi, and I. Hajnsek, "A polarimetric temporal scene parameter and its application to change detection," in Proc. IEEE Int. Geosci. Remote Sens. Symp., IGARSS 2011, Vancouver, Canada, 24-29 July 2011, pp. 1091-1094.

[8] X.-V. Phan, L. Bombrun, G. Vasile, and M. Gay, "Heterogeneous clutter models for change detection in PolSAR imagery," in Proc. POLinSAR 2011, Frascati, Italy, vol. ESA SP-695, 7 pp., Mar. 2011.

[9] K.C.S. Pillai and P. Samson, Jr., "On Hotelling's generalization of $T^{2}$," Biometrika, vol. 46, no. 1/2, pp. 160168, June 1959.

[10] David T. Hughes and John G. Saw, "Approximating the percentage points of Hotelling's generalized $T_{0}^{2}$ statistic," Biometrika, vol. 59, no. 1, pp. 224-226, Apr. 1972.

[11] J.J. McKeon, "F approximations to the distribution of Hotelling's $T_{0}^{2}$," Biometrika, vol. 61, no. 2, pp. 381383, Aug. 1974.

[12] S.N. Anfinsen and T. Eltoft, "Application of the matrixvariate Mellin transform to analysis of polarimetric radar images," IEEE Trans. Geosci. Remote Sens., vol. 49, no. 6, pp. 2281-2295, June 2011.

[13] J. Kittler and J. Illingworth, "Minimum error thresholding," Pattern Recognition, vol. 19, no. 1, pp. 41-47, Jan. 1986.

[14] G. Moser and S.B. Serpico, "Generalized minimumerror thresholding for unsupervised change detection from SAR amplitude imagery," IEEE Trans. Geosci. Remote Sens., vol. 44, no. 10, pp. 2972-2982, Oct. 2006.

[15] S.N. Anfinsen and V. Akbari, "Statistical characterisation of the complex hotelling-lawley trace statistic," IEEE Trans. Geosci. Remote Sens., 2013, submitted.

[16] P. Graczyk, G. Letac, and H. Massam, "The complex Wishart distribution and the symmetric group," Ann. Statist., vol. 31, no. 1, pp. 287-309, Feb. 2003.

[17] W.C. Parr and W.R. Schucany, "Minimum distance estimation and components of goodness-of-fit statistics," J. Royal Statist. Soc., ser. B, vol. 44, no. 2, pp. 178-189, 1982.

[18] S.N. Anfinsen, A.P. Doulgeris, and T. Eltoft, "Estimation of the equivalent number of looks in polarimetric synthetic aperture radar imagery," IEEE Trans. Geosci. Remote Sens., vol. 47, no. 11, pp. 3795-3809, Nov. 2009. 